\title{
TRATAMIENTO DE ORTODONCIA 4x2 EN UN PACIENTE CON ENFERMEDAD PERIODONTAL.
}

\section{4x2 ORTHODONTICS TREATMENT IN A PATIENT WITH PERIODONTAL DISEASE.}

\author{
Miriam Fernanda Ortega López ${ }^{1}$, Ronald Roossevelt Ramos Montiel $^{2}$, Jenny Fabiola Anchundia Reinoso ${ }^{3}$, Santiago \\ Daniel Serrano Piedra ${ }^{4}$, Diego Xavier Toral Aguilera ${ }^{5}$ \\ ${ }^{1}$ Especialista en Docencia Clínica. Cursante del Posgrado en Odontopediatría Universidad UTE Quito- Ecuador. \\ ${ }^{2}$ Especialista en Ortodoncia. Cursante del doctorado de Ciencias Odontológicas de la Universidad de Zulia Venezuela. \\ Catedrático de la carrera de Odontología de la Universidad Católica de Cuenca. \\ ${ }^{3}$ Rotante de Investigación. Carrera de Odontología de la Universidad Católica de Cuenca, Ecuador. \\ ${ }^{4}$ Especialista en Estética Dental. Catedrático de la Carrera de Odontología de la Universidad Católica de Cuenca, Ecuador. \\ ${ }^{5}$ Especialista en Periodoncia e Implantología. Catedrático de la Carrera de Odontología de la Universidad Católica de \\ Cuenca, Ecuador. \\ *mfortegal38@ hotmail.com
}

\begin{abstract}
Resumen
La correlación entre varios campos de la odontología esta determinada por un tratamiento multidisciplinario debido a que pacientes que presentan enfermedad periodontal en la mayoría de los casos acuden al odontólogo con la necesidad de un tratamiento estético y la ortodoncia puede ser la solución de obtenerlo, teniendo en cuenta que primero se resolverá el problema de salud periodontal, para cumplir la efectividad en el tratamiento orto-perio en aquellos pacientes con enfermedad periodontal es analizar el efecto que produce el tratamiento ortodóncico sobre el periodonto, la reacción de fuerzas que se pueden utilizar, la situación periodontal del paciente que necesitan esta clase de tratamientos y las consideraciones frente al paciente que no posee los recursos económicos suficientes para realizarlos. En este caso clínico se examinó a un paciente de 29 años de edad con limitación de recursos económicos para realizarse un tratamiento odontológico, se realiza mediante el análisis de una secuencia de diagnósticos y tratamientos multidisciplinarios y así poder alcanzar los objetivos primordiales del paciente.
\end{abstract}

Palabras clave: Tratamiento multidisciplinario, ortodoncia, estética, planificación, tejidos periodontales..

\begin{abstract}
The correlation between various fields of dentistry is determined by a multi-disciplinary treatment because patients who present periodontal disease in most cases go to the dentist with the need for aesthetic treatment and orthodontics may be the solution to obtain it, taking into account that first the periodontal health problem will be solved, to comply with the effectiveness of ortho-periodontal treatment in those patients with periodontal disease is to analyze the effect produced by orthodontic treatment on the periodontium, the reaction of forces that can be used, the periodontal situation of the patient who needs this kind of treatment and the considerations against the patient who does not have sufficient economic resources to perform them. In this clinical case, a 29-year-old patient with limited economic resources was examined for dental treatment. This was done by analyzing a sequence of multidisciplinary diagnoses and treatments in order to achieve the patient's primary objectives.
\end{abstract}

Key words: Multidisciplinary treatment, orthodontics, aesthetics, planning, periodontal tissues.

\section{INTRODUCCIÓN}

La ortodoncia es una rama de la odontología que proviene de dos vocablos griegos ortho: recto y donto: diente, en la actualidad se podrían describir un sin número de clasificaciones o tipos de tratamientos; sin embargo, se podría generalizar su tratamiento en tres etapas principales: la preventiva como su nombre lo indica, ayudará a prevenir maloclusiones, la interceptiva es aquella que capta un problema instaurado lo intercepta y lo corrige siempre y cuando se encuentre dentro de las etapas del crecimiento cráneo maxilo; y finalmente la ortodoncia correctiva, que al igual que la ortodoncia interceptiva capta un problema instaurado lo intercepta y lo corrige pero cuando no existe un crecimiento de las bases óseas. ${ }^{12}$ 
El profesional de ortodoncia debe estar capacitado para valorar de manera multidisciplinaria a pacientes con distintas patologías o alteraciones en el sistema estomatognático; por lo tanto, no es solo tratar una maloclusión o corregir distintos grados de apiñamiento, es realizar un tratamiento integral con la ayuda de las distintas áreas de las especialidades odontológicas. La enfermedad periodontal en cualquiera de sus etapas es sin duda uno de los principales signos clínicos en el apiñamiento dental junto con la caries, por lo tanto, no solo se trata de mejorar la estética sino la función, higiene e interacción de todos los componentes del sistema estomatognático. $^{3}$

El presente caso clínico se enfoca en el tratamiento ortodóntico correctivo, en el cual se requiere el uso de aparatología fija para corregir mal posiciones dentarias. Dentro de los objetivos completos del tratamiento se espera mejorar la posición de las piezas dentales alteradas, pudiéndose colocarse brackets en toda la arcada dentaria o en grupos de dientes como por ejemplo la técnica $4 \times 2$. Durante un tratamiento ortodóntico se presentan etapas tales como alineación, nivelación y corrección de rotaciones, cierre de espacios y control de línea media y torque radicular, asentamiento y contención; las etapas mencionadas pueden variar según la filosofía, prescripción o criterio del profesional ${ }^{4}$

$\mathrm{Al}$ aplicar una fuerza en un grupo de dientes o en uno se producirá una alteración en su estado de equilibrio causando movimientos dentales bajo la acción de fuerzas y al perder la actividad producirá un estado de descanso. ${ }^{5,6}$ la fuerza aplicada va de $20-25 \mathrm{~g}$ por $\mathrm{cm} 2$ o menos de $80 \mathrm{~g}$ por $\mathrm{cm} 2$, si deseamos inclinar una pieza dental la fuerza aplicada es de $50 \mathrm{~g} / \mathrm{cm} 2$ en la superficie labial de un incisivo, si se desea el movimiento del diente hacia lingual la fuerza aplicada será de $12.5 \mathrm{~g} / \mathrm{cm} 2 .^{7}{ }^{8}$ para cerrar un diastema se producirá fricción utilizando alambres rectangulares de acero inoxidable 0,019 $\mathrm{x} 0,025$, en brackets con ranuras 0,022 x 0,028 y 0,016 $\mathrm{x} 0,022$, en ranuras $0,018 \times 0,025$ permitiendo un control bucolingual y manteniendo la angulación de los dientes, pero esta no es recomendada a nivel de caninos y premolares pero si en el sector anterior. ${ }^{910}$

Fuerzas aplicadas por los brackets, alambres y elásticos en los dientes que se desea mover no deben exceder la capacidad de respuesta biológica del paciente, para no provocar movimientos irreversibles o reabsorciones radiculares a los que se le clasifica de la siguiente manera según sus efectos biológicos, primer grado: movimientos leves y rápidos que no afectan al periodonto, segundo grado: fuerzas menor a la presión sanguínea en el ligamento periodontal con disminución en el aporte sanguíneo al periodonto va a producir reabsorción ósea en el área de la presión cuando este cesa el periodonto regresa a su estado normal sin reabsorción radicular $^{113},{ }^{12}$ tercer grado: fuerza superior a la presión sanguínea va a originar isquemia por compresión del ligamento periodontal y el cuarto grado: aplicación de fuerzas extensas producirán reabsorción radicular.
Los tejidos que conforman la cavidad oral son aquellos que están formados por periodonto de unión y de protección los mismos que están fuertemente relacionados, debido a que la estructura principal que es periodonto une todas las estructuras de soporte dental, como a la mucosa masticatoria, es por ello, cuando algún tejido enferma, los demás no cumplen sus funciones adecuadas. ${ }^{313}$

El grado de enfermedad periodontal, inflamación y pérdida de inserción se relaciona con la edad del paciente, presentando clínicamente pérdida de las papilas interdentales, márgenes gingivales, diastemas, los mismos que causaran molestias estéticas y funcionales al paciente es por eso que muchos de ellos acuden a tratamientos ortodónticos. ${ }^{14},{ }^{12}$ pero para que este tratamiento presente resultados favorables va a depender de otros factores entre ellos la higiene bucal, presentando un mejor control de placa dentobacteriana o biofilm en un paciente adulto comparado a un adolescente, considerando que esta patología no es muy frecuente en adolescentes, pero debido al recambio dental y cambios hormonales va a potenciar los procesos inflamatorios. ${ }^{315}$

Es de gran importancia que al comenzar un tratamiento ortodóntico se realice un diagnostico periodontal completo con el objetivo de evitar efectos indeseables ocasionados por los movimientos ortodónticos en pacientes con enfermedad periodontal no tratada, teniendo mayor cuidado en el sangrado ya que es la evidencia de actividad periodontal, al aplicar ortodoncia va a existir presión en el ligamento periodontal provocando una disminución en el aporte sanguíneo, si el paciente no presenta un correcto soporte óseo este no impedirá la realización del tratamiento ortodóntico con un adecuado control periodontal. ${ }^{12163}$

Se ha podido demostrar que el movimiento dental en defectos óseos, como la extrusión, intrusión ortodóncica y la inclinación labial de piezas dentales anteriores puede realizarse sin afectar el periodonto de soporte siempre y cuando exista un buen control de placa y detartraje gingival cada 2 semanas dependiendo del caso con el cual vamos a prevenir un desplazamiento coronal de la encía disminuyendo la recidiva. ${ }^{17}$

Se describirá el tratamiento de los tejidos blandos periodontales en diferentes etapas, antes, durante y después del tratamiento ortodóntico, empleando técnicas periodontales sencillas con las que se podrá conseguir mejor nivel de función y estética de los tejidos blandos en los pacientes sin enfermedad periodontal o en pacientes con patologías periodontales que estén recibiendo o han recibido tratamiento ortodóncico. $^{3}$

\section{REPORTE DE CASO}

Acude a la Clínica Integral de la Facultad de Odontología de la Universidad Católica de Cuenca, sede en Cuenca, un paciente de sexo masculino de 29 años de edad por problemas de estética al cual se le planteo la realización de rehabilitación oral integral. El paciente no presentaba ninguna enfermedad 
que requerían cuidados especiales para la ejecución de su tratamiento (ASA1). Refería que tenía hábitos nocivos de tabaco, de dos veces por semana.

Al examen clínico intraoral se presentó, diastema de los dos incisivos centrales con giroversión de las mismas, pieza dentaria 4.1 con cálculo supra y subgingival y recesión de clase 1 Miller. (Fig.1), en el maxilar superior presencia de lesiones cariosas en piezas posteriores y ausencia de piezas dentarias posteriores. (Fig. 2), en el maxilar inferior presento recesión gingival en piezas dentarias anteriores y ausencia de piezas dentarias posteriores. (Fig.3)

Al examen radiográfico se pudo observar que tanto en el maxilar superior e inferior presentaban reabsorción ósea horizontal generalizada, ausencia de piezas dentarias posteriores y mesioangulación de pieza dentaria 3.7. (Fig.4)

En el análisis de modelos de estudio ortodóntico en vista frontal y en oclusión se pudo observar una mordida profunda, la línea media no era coincidente con la de la arcada, diastemas de piezas centrales anteriores. (Fig.5) En el análisis de modelos de estudio ortodóntico en Vista lateral derecha e izquierda se pudo observar un Overjet: $5 \mathrm{~mm}$ y un Overbite: $4 \mathrm{~mm}$ (mordida profunda), relación molar: Derecha: Clase 1, izquierda: no registrable, relación canina: Derecha: clase 3 e Izquierda clase 2. (Fig.6)

Estableciendo el siguiente diagnóstico: desviación de la línea media, diastema y apiñamiento dental, edentulismo parcial, migración dental, periodontitis crónica moderada localizada y presencia de lesiones cariosas.El plan de tratamiento consistió en: una fase preventiva (técnicas de higiene). Se realizó tratamiento periodontal que incluyó profilaxis, detartraje supra y subgingival y se le indicó el uso se clorhexidina al $0.12 \%$ por 15 días. (Fig.7)

La segunda etapa, se colocó Ortodoncia técnica $4 \times 2$ para realizar movimiento de dientes anteriores aplicando la nivelación parcial hasta caninos permitiendo que colocándolos en estos tengamos mayor control en movimiento de giro de incisivos. (autor) Se aplicaron alambres de bajo calibre en la primera sesión (0,012 milésimas de pulgadas), con una fuerza de 10 a 20 gr debido a que el paciente presenta pérdida ósea.

Se aplicó fuerza cupla a las que se les define como "fuerzas iguales o reciprocas que van en diferentes direcciones", ${ }^{18}$ esta fuerza se aplicara a nivel de los incisivos. Se utilizó botones en las caras palatinas de las piezas $1.1 \mathrm{y}$ 2.1, Finalmente se realizó ajuste oclusal. (Fig.8)En La tercera etapa se realizó la Endodoncia en piezas dentarias 2.5, 3.4 para su posterior rehabilitación. (Fig.9)

La cuarta etapa se enfocó en la rehabilitación oral. Se realizó las restauraciones de las lesiones cariosas. En el sector que presentaba ausencia de piezas dentarias se realizó: prótesis fija de las $2.5,2.6$ y 2.7 y prótesis removible de las piezas 3.4 , 3.5, 3.6, y la colocación de carillas de resina en las piezas dentales anteriores. (Fig.10), (Fig.11), (Fig.12).

\section{DISCUSIÓN}

El presente artículo destacó la importancia del tratamiento multidisciplinario en el paciente adulto se trabajó el área de la periodoncia con detartraje supra y subgingival, y la motivación de un resultado exitoso con una buena higiene bucal. ${ }^{9}$

La planeación del tratamiento ortodóncico para este tipo de maloclusión fue utilizar una aparatología fija parcial o la denominada técnica $4 \times 2$ y así reducir el tiempo de tratamiento en comparación de un tratamiento con aparatología completa. En esta técnica se usaron brackets en los dientes anteriores y primeros molares generando un adecuado sistema de fuerzas. Y por último con el restaurador para mejorar la salud, función y estética del paciente. ${ }^{9}$

El propósito de realizar un tratamiento de Ortodoncia en pacientes con enfermedad periodontal fue mejorar la salud, función y estética del paciente, el paciente no presentó trastornos periodontales después del tratamiento ortodóncico debido a su buena higiene oral. En los estudios realizados según Tortolini y Fernández determinaron que los pacientes con enfermedades periodontales se benefician del objetivo de sus movimientos dentales a partir de un tratamiento ortodóncico si su enfermedad periodontal se encuentra inactiva. ${ }^{19}$

Cuando el paciente presenta una la higiene bucal deficiente existen complicaciones al realizar un tratamiento ortodóntico por la presencia de inflamación o sangrado que dificulta observar el éxito en el tratamiento, en el estudio de un paciente de enfermedad periodontal que fue sometido a tratamiento de ortodoncia por Millán et al en 2007, explica que no logró buenos resultados debido a que no hubo colaboración o motivación por parte del paciente; sus malos hábitos de higiene dificultaron la colocación de la aparatología ortodóncica. ${ }^{20}$

Según Van Gastel y cols. 2008 y Barbieri y cols. 2013, el tratamiento ortodóncico es considerado como un factor de riesgo de enfermedad periodontal por el difícil acceso de higiene bucal, si el paciente no colabora en su higiene puede facilitar el acumulo de bacterias hasta una posible reabsorción ósea. ${ }^{21}$

Durante el tratamiento se recomienda al paciente exámenes radiográficos una vez al año que pueda servir para la detección de caries y la observación de reabsorción radicular. Después de terminar el tratamiento ortodóntico según Mathews et al en el año 2005, el paciente debe recibir mantenimiento periodontal dependiendo del grado ya sea cada dos o tres meses y así exista un adecuado remodelamiento óseo, disminuya la movilidad y se remodelen los ligamentos periodontales, además recomienda una evaluación radiográfica cada seis meses o una vez al año. ${ }^{20}$

Los pacientes sometidos a tratamientos ortodónticos con enfermedad periodontal pueden llegar a tener buenos resultados, ya que en los últimos estudios se ha demostrado el éxito de trabajar de una manera multidisciplinaria. Estudios demuestran que el tratamiento ortodóntico distribuye 
fisiológicamente fuerzas oclusales sobre el periodonto, sin embargo, pacientes que no cumplan con parámetros como la adecuada higiene bucal antes de empezar con un tratamiento de ortodoncia podría llevar al fracaso. Por eso es necesario realizar seguimiento al paciente y mantenimiento periodontal durante un lapso de tiempo para no generar complicaciones, beneficiándole así al paciente una sonrisa que cambie su vida.

\section{ANEXOS}

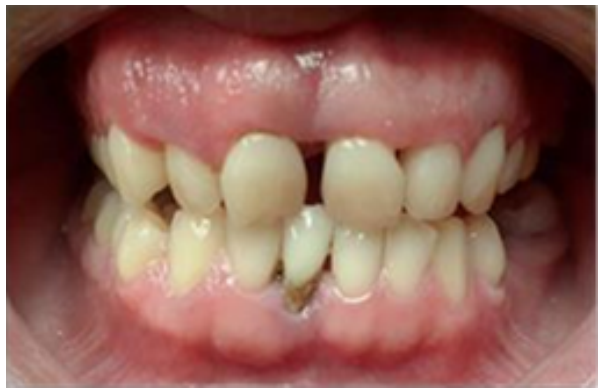

Fig. 1: Fotografía intraoral de frente, presencia de diastema y cálculo supra y subgingival.

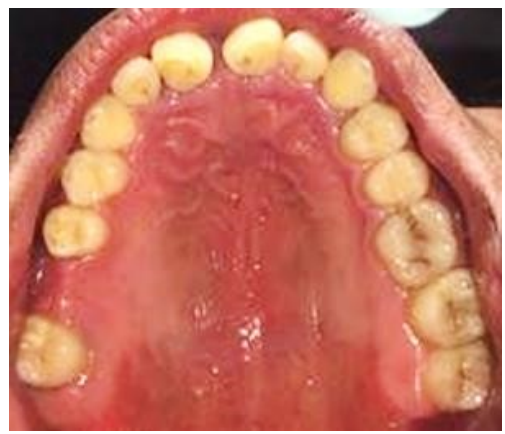

Fig. 2: Fotografía intraoral del maxilar superior con presencia de lesiones cariosas, y edentulismo parcial.

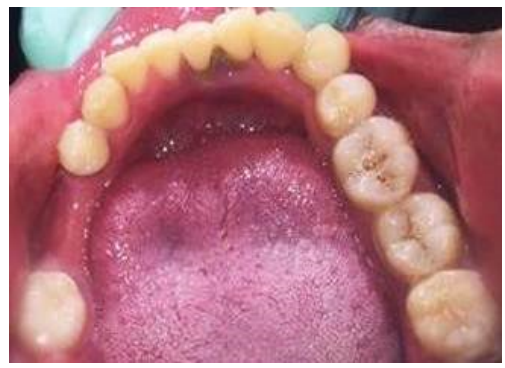

Fig. 3: Fotografía intraoral del maxilar inferior: presencia de lesiones cariosas y edentulismo parcial.

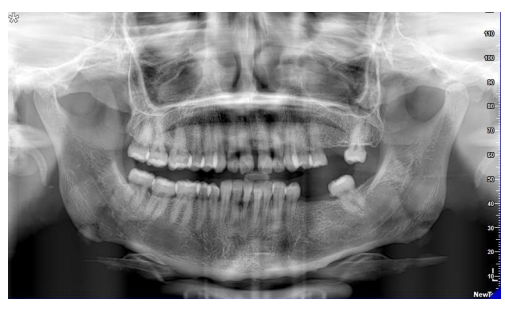

Fig. 4: Radiografía panorámica, maxilar superior e inferior con reabsorción ósea horizontal y edentulismo parcial.

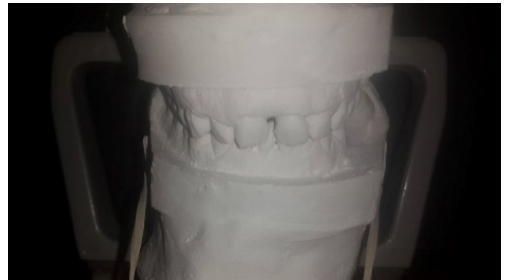

(a) Vista frontal de los modelos de estudio en oclusión.

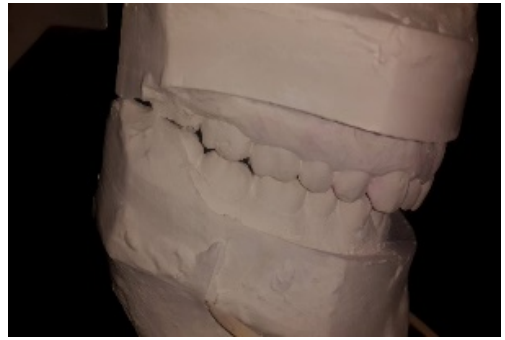

(b) Vista lateral derecha de los modelos de estudio.

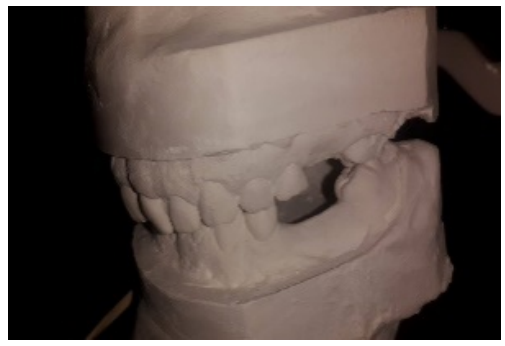

(c) Vista lateral izquierda de los modelos de estudio.

Fig. 5: Análisis de Modelos de Estudio de Ortodoncia

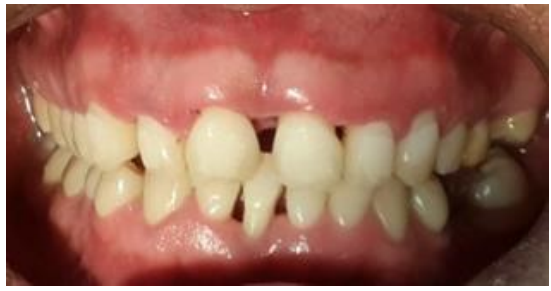

Fig. 6: Fotografía intraoral de frente: Fase preventiva. 


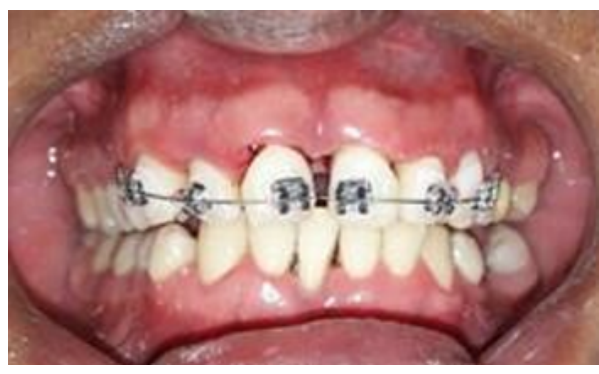

Fig. 7: Fotografía intraoral de frente: Segunda etapa, tratamiento ortodóntico con técnica $4 \times 2$
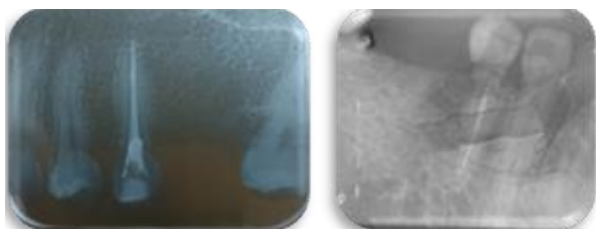

Fig. 8: Exámenes radiográficos intraorales: Tercera etapa, tratamiento endodóntico para su posterior rehabilitación.

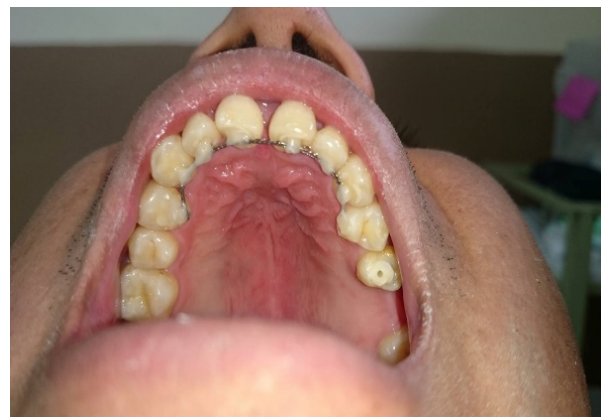

(a) Maxilar superior, restauración de lesiones cariosas.

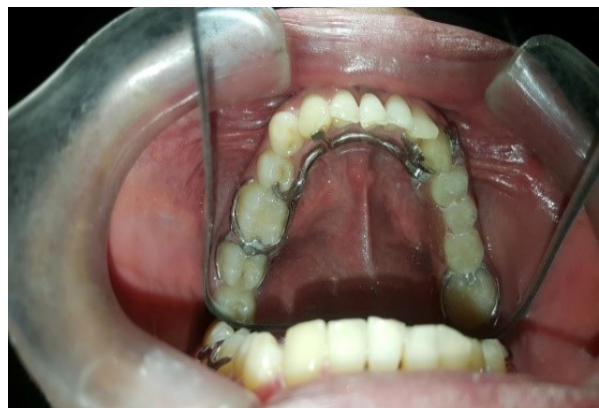

(b) Maxilar Inferior Prótesis removible.

Fig. 9: Fotografías intraorales: Cuarta etapa, rehabilitación

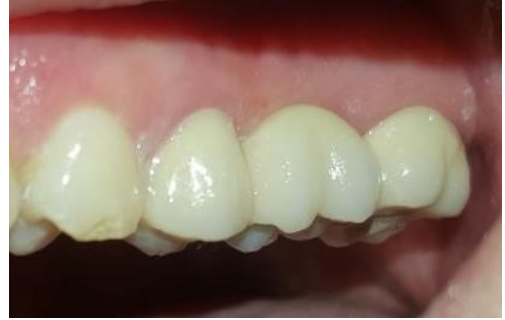

Fig. 10: Fotografía intraoral superior izquierda: Cuarta etapa, rehabilitación oral: prótesis fija superior.

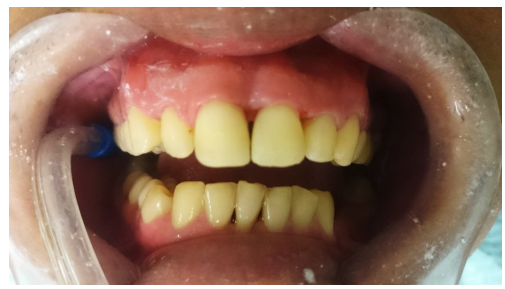

Fig. 11: Fotografía intraoral de frente: Cuarta etapa, rehabilitación oral: Carillas de resina.

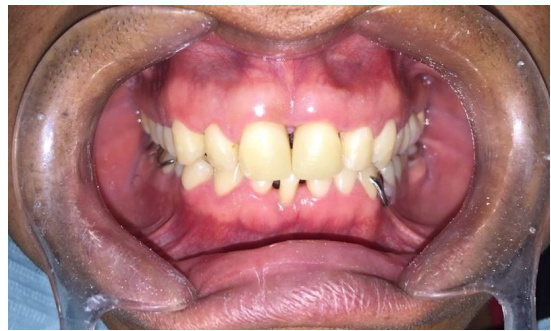

Fig. 12: Fotografía intraoral de frente: Tratamiento multidisciplinario finalizado.

\section{Conflicto de Intereses}

Los autores declaran no tener conflicto de intereses.

\section{Referencias Bibliográficas}

1 Rodriguez JGT. Etiologias de Chile. [Online].; 2020 [cited 202007 25. Available from: http://etimologias.dechile.net/?ortodoncia.

2 Quirós O. Introducción a la Ortodoncia. Acta Odontológica Venezolana. 2004 Sep; 4(3).

3 Tortolini P FBE. Ortodoncia y periodoncia. Avances en Odontoestomatología. 2011 Mar; 27(4): p. 10.

4 A. Cervera Sabater MSP. Ortocervera. [Online]. Available from: http://ortocervera.com/alineacion-y-nivelacioncon-arco-recto-biomecanica-basica/.

5 Uribe RGA JMJF. Principios de física que se aplican en ortodoncia. In Restrepo GAU, editor. Principios de física 
que se aplican en ortodoncia. Colombia: CIB Corporacion para Investigacion Biologica; 2010. p. 1312.

6 Balaguer. VO. Movimiento dentario. Gaceta Dental. 2009 Feb.

7 Laguiadelprotesico. [Online]; $2020 \quad$ [cited $2020 \quad 0326 . \quad$ Available from: https://www.laguiadelprotesico.site/biomecanica-enortodoncia/.

8 T. Kohno YMZk. Movimiento dental experimental bajo ligeras fuerzas de ortodoncia: tasas de movimiento dental y 17 cambios del periodonto. Journal of Orthodontics. 2001 Feb; 29(2).

9 Coa P. Friccón en Ortodoncia. Rev. Evid. Odontol. Clinic. $2016 \mathrm{Jul} ; 2$ 2(2).

10 Pamela RR, Valeria SC, Estuardo BC. Cierre de espacios 19 con fricción - Revisión de Literatura. Revista Latinoamericana de Ortodoncia y Odontopediatría. 2014 Jul.

11 Harfin JFd. Tratamiento Ortodóntico en el Adulto. In Alvear 20 Porto II. Tratamietno ortodóntico en paciente con periodonMT, editor. Tratamiento Ortodóntico en el Adulto. Buenos Aires: Panamericana ; 2005. p. 384 páginas.

12 Sanz DM. Las relaciones entre la ortodoncia y la periodoncia. Gaceta Dental. 2012;: p. 3.

13 Lindhe J, TKyMA. Anatomía de los tejidos periodontales. In Lindhe J. Periodontología Clínica e Implantología Odontología. Argentina: Editorial Médica Panamericana; 2006. p. 1408 .

14 Ortiz AVO, EGCM. Tratamiento Ortodontico del paciente perdiontal con pérdida severa de insercción. Periodoncia Clínica. 2016 Jun;: p. 8.

5 Peña CPR. Consecuencias periodontales después del tratamiento de ortodoncia en pacientes adultos con apiñamiento severo. Revista Latinoamericana de Ortodoncia y Odontopediatría. 2017 Sep.

16 Sada GV, CRG. Enfoque ortodóncico en el tratamiento multidisciplinario de pacientes adulto $\mathrm{Su}$ relación con la periodoncia. RCOE. 2003; 8(6): p. 12

17 Bueno DL. Ortodoncia y Periodoncia, Dos Especialidades que van de la mano. Fundación Juan José Carraro. 2014 Jul.

18 Sapienza GAV, DBGMACA. Sistemas de cupla y arco transpalanace modificado para desrotación de premolares. Revista de Ortodoncia y Odontopediatría. 2013.

Russell D LP. Orthodontics Treatmente in an adult patient with inactive periodontal disease. Revista Mexicana de Ortodoncia. 2016 Mar; 4(1): p. 4. titis crónica avanzada generalizada: reporte de un caso. Acta Odontologica Colombiana. 2016; 6(1): p. 6.

21 Ortiz A GE. Tratamiento ortodóntico del paciente periodontal con pérdida avanzada de inserción: Artículo de revisión. Dialnet. 2016.

Recibido: 12 de Abril del 2020

Aceptado: 25 de Agosto del 2020 